Neuromolecular Med. 2010 December ; 12(4): 301-305. doi:10.1007/s12017-010-8135-5.

\title{
Sphingolipids in Neurodegeneration
}

\author{
Norman J. Haughey \\ Department of Neurology, Richard T. Johnson Division of Neuroimmunology and Neurological \\ Infections, The Johns Hopkins University School of Medicine, Meyer 6-109, 600 North Wolfe \\ Street, Baltimore, MD 21287, USA
}

\section{Abstract}

Although the brain contains a high content of sphingolipids, we know relatively little about the roles that sphingolipids play in regulating neural functions. Once regarded only for their structural roles in maintaining the integrity of cellular and sub-cellular compartments, it is now apparent that many sphingolipid species are biologically active and play important roles in regulating signaling events. Recent technological and scientific advances are rapidly increasing our knowledge of the roles that sphingolipids play in regulating normal neural activity. Likewise, we are beginning to understand how perturbations in sphingolipid metabolism contribute to the pathogenesis of a variety of neurodegenerative conditions. In this special issue of NeuroMolecular Medicine, we present a series of review articles that summarize new and emerging technologies for the analysis of sphingolipids, sphingolipid metabolic pathways, and how dysfunctions in sphingolipid metabolism contribute to neurodegeneration in lysosomal storage disorders, Alzheimer's disease and Multiple Sclerosis.

\section{Keywords}

Sphingolipid; Sphingomyelin; Ceramide; Neurodegeneration; Alzheimer's; HIV; Multiple sclerosis; Neurodegeneration; Synapse

\section{Overview}

The last decade has seen rapid advances in our understanding of lipid biology. In particular, there have been great strides in our knowledge of the mechanisms by which sphingolipids regulate numerous cellular processes. These discoveries have been possible in part due to technological advances that allow for the identification and quantitative analysis of individual sphingolipid species, advances in basic research that continue to map the metabolic pathways which regulate sphingolipid metabolism and an increased understanding that dysregulated sphingolipid metabolism plays important roles in the pathogenesis of a number of different neurodegenerative conditions. The brain is rich in lipids and has a high content of sphingolipids. It is therefore not surprising that many recent discoveries pertaining to sphingolipid biology have focused on understanding the involvement of this class of lipids in basic neuronal and glial processes, and how perturbations in sphingolipid

(C) Springer Science+Business Media, LLC 2010

N. J. Haughey nhaughe1@jhmi.edu. 
metabolism contribute to neurodegenerative disease. In this special issue of NeuroMolecular Medicine are a series of review articles that summarize our current knowledge of roles for dysregulated sphingolipid metabolism in neurodegeneration. Chen et al. summarize our current understanding of modern technologies for the discovery and quantitative analysis of individual lipid species and sphingolipid metabolic pathways. Wu et al. provide a focused review of neutral sphingomyelinase signaling with an emphasis on pathways implicated in neurodegeneration. Eckhardt provides a review of disturbances of sphingolipid metabolism that occurs in lysosomal storage diseases, including current and developing therapies for this group of disorders. Jana \& Pahan provide a comprehensive review of roles for dysfunctional sphingolipid metabolism in dysregulating immunological and neural functions in the setting of Multiple Sclerosis. Mielke \& Lyketsos review our current understanding of how sphingolipid metabolism is dysregulated in brains of patients with Alzheimer's disease, with an emphasis on the potential utility of blood sphingolipids as biomarkers for cognitive status. This preface is intended to provide the reader with a brief introduction to some of the known roles for sphingolipids in regulating neuronal and glial functions in order to highlight potential systems that may be perturbed when sphingolipid metabolism is altered in the setting of a neurodegenerative disease.

\section{Mechanisms for Targeting}

Over half of all proteins are located in or near membranes and the lipid environment surrounding these proteins plays critical roles in regulating the function of these membraneassociated proteins. It is therefore not surprising that a number of conserved lipid-binding domains have been identified in eukaryotes. Thus far, at least 11 lipid-binding domains have been identified that include $\mathrm{C} 1$ and $\mathrm{C} 2$ (conserved regions among PKC isoforms with distinct lipid-binding properties), plekstrin homology $(\mathrm{PH})$ domains that bind to phosphatidylinositols (and to other proteins), zinc finger FVYE domains that bind phosphatidylinositol 3-phosphate, Phox homology (PX) domains that bind phosphatidylinositol(4,5)P2 and phosphatidylinositol(1,4,5)P3, Epsin N-terminal homology (ENTH) and AP180 N-terminal homology (ANTH) domains that bind phosphatidylinositol(4,5)P2, Bin-Amphiphysin-Rvs (BAR) domains that bind to concave shapes in membranes, 4.1 protein ezrin/radixin/moesin (FERM) domains that bind inositol(1,4,5)P3 and phosphatidylinositol 4,5-bisphosphate, PDZ (named after the first proteins discovered to share the domain - postsynaptic density protein, Drosophila disk large tumor suppressor and zonula occludens-1 protein) and TUBBY domains that bind phosphatidylinositols (Medkova and Cho 1999; Harlan et al. 1994; Kutateladze et al. 1999; Stahelin et al. 2003; Blood and Voth 2006; Hirao et al. 1996; Santagata et al. 2001). Although motifs that specifically target proteins to ceramides or other sphingolipids have not yet been identified, a recent study identified the C3 domain of Kinase Suppressor of Ras 1 as a lipid-binding moiety that effectively binds ceramide but not other lipids including 1,2diacylglycerol, dihydroceramide, ganglioside GM1, sphingomyelin and phosphatidylcholine (Yin et al. 2009). In addition to these known protein motifs, (GPI) anchors are known to facilitate the targeting of proteins to membranes by increasing hydrophobicity and thereby promoting membrane targeting, and there are a number of protein modifications including acylation, myristoylation, prenylation, palmitoylation that promote membrane localization 
and are involved in protein-protein interactions (Low 1989; Levental et al. (2010); Magee et al. 1989; Thelen et al. 1991; Stokoe et al. 1994; Liu et al. 1993). Thus, one mechanism by which perturbations in sphingolipid metabolism may contribute to dysfunctional signaling in neurodegenerative conditions is by contributing to the mislocalization of proteins.

\section{Membrane Microdomains}

Perturbations in cellular signaling have been reported in virtually every neurodegenerative disease. Spatial and temporal features of cellular signaling are in part controlled by lipid components that can regulate protein location and scaffolding events through a dynamic modulation of membrane microdomains. The lipid-raft-enriched sphingolipids ceramide and sphingosine are regarded as important regulators of synaptic functions and have been shown to play important roles in synapse formation, neurotransmitter release and synaptic plasticity (Wheeler et al. 2009; Yang 2000; Brann et al. 1999; Ping and Barrett 1998; Inokuchi et al. 1998; Furuya et al. 1998; Furukawa and Mattson 1998; Ito and Horigome 1995). For example, the application of synthetic cell-permeable analogs of ceramide to hippocampal slice cultures increased excitatory postsynaptic currents, but did not modify paired-pulse facilitation (Furukawa and Mattson 1998; Coogan et al. 1999; Fasano et al. 2003), suggesting that ceramides can exert biological effects at postsynaptic sites. These ceramideinduced enhancements of excitatory currents were typically transient and followed by a sustained depression of excitatory postsynaptic currents (Coogan et al. 1999; Yang 2000; Furukawa and Mattson 1998; Tabarean et al. 2006; Davis et al. 2006), perhaps due to endogenous regulatory mechanisms that limit the biological effects of ceramide in postsynaptic terminals. One mechanism by which sphingolipids regulate activity at postsynaptic sites involves the formation of specialized membrane microdomains. These lipid microdomains (often referred to as lipid rafts) play important roles in regulating cellular signaling through protein-lipid interactions and by influencing the physical properties of the membrane. Lipids rafts are highly ordered membrane domains enriched in sphingomyelin, ceramide, gangliosides and cholesterol. The high degree of saturated bonds in raft-associated lipids increases packing density and reduces lateral mobility, when compared with regions of the bilayer containing more unsaturated lipids. The conformational shape of the amide and hydroxyl groups in $\mathrm{C} 3$ region of ceramides further stabilizes these microdomains by allowing for the establishment of a series of hydrogen bonds that further stabilize these microdomains. However, it should be noted that not all components of lipid rafts interact equally well, suggesting that there may be a lateral organization of components within lipid rafts and/or sub-types of membrane microdomains that differ in their primary lipid components. Nevertheless, these microdomains appear to selectively recruit and/or sequester signaling molecules to organize signaling units that regulate the coupling of extracellular signals to intracellular signal transduction machinery. Thus, a disturbance in sphingolipid composition could contribute to neuronal dysfunction and neurodegeneration by disrupting spatial and temporal controls for cellular signaling. 


\section{Regulation of Synaptic Activity by Sphingolipids}

Dysfunctions in synaptic activity and the loss of synaptic connections play pivotal roles in neurodegeneration. Accumulating evidence suggests that sphingolipids are involved in the regulation of synaptic strength at both presynaptic and postsynaptic sites.

\section{Postsynaptic Mechanisms}

Membrane microdomains have been implicated in the regulation of synaptic activity (Stahelin 2009; Day and Kenworthy 2009; Swartz 2008; Owen et al. 2009). Brief and controlled modifications in membrane components that alter the biophysical properties of membranes can regulate the traffic and surface expression of transmembrane receptors (Stahelin 2009; Day and Kenworthy 2009; Swartz 2008; Owen et al. 2009; Wheeler et al. 2009). For example, NMDA receptors have been shown to traffic to lipid rafts where transient modifications in diacylglycerol and ceramide alter the physical properties of the bilayer to promote the fusion of receptor-laden vesicles with the plasma membrane (Wheeler et al. 2009; Bandaru et al. 2009; Bandaru et al. 2007; Haughey et al. 2004). Approximately $60 \%$ of NMDA receptors are located in lipid rafts (Besshoh et al. 2005; Fullekrug and Simons 2004), and these receptors can be induced to traffic in or out of lipid rafts with important implications for signal transduction, synaptic plasticity and cell survival (DelintRamirez et al. 2010). For instance, disrupting lipid rafts by removal of cholesterol from membranes inhibits NMDA receptor currents and calcium flux and increases the basal internalization rate of AMPA receptors (Hering et al. 2003; Frank et al. 2004; Abulrob et al. 2005). Although the mechanisms that regulate lateral movement of receptors through the bilayer are largely unknown, they appear to involve physical and electrostatic interactions between proteins with particular lipids. It is possible that post-translational modifications such as phosphorylation that alter the conformational shape of proteins could modify the amino acid residues which line the protein-lipid interface to favor interactions with particular lipids. However, it has not been clearly shown if protein phosphorylation occurs in lipid rafts or if proteins associate with lipid rafts after they are phosphorylated (AssaifeLopes et al. (2010); Bryant et al. 2009; Wheeler et al. 2009).

\section{Presynaptic Mechanisms}

At the presynaptic terminal, the fusion of synaptic vesicles with plasma membrane appears to require focal changes in lipid content that prepare the vesicle to dock with the membrane. The docking and fusion of synaptic vesicles with the plasma membrane is regulated via a conserved family of soluble $N$-ethylmaleimide-sensitive factor activating protein receptor (SNARE) proteins including synaptobrevin that is located to synaptic vesicles and syntaxin and SNAP-25 that are located to the plasma membrane. Normally, synaptobrevin adheres tightly to synaptic vesicles through electrostatic and hydrophobic interactions. The creation of sphingosine in synaptic vesicle membranes neutralizes electrostatic interactions of synaptobrevin with phospholipids, allowing synaptobrevin to interact with syntaxin/ SNAP-25 (Darios et al. 2009). Presynaptic activation of ceramidase (catalyzes the deacylation of ceramide to produce a free fatty acid and sphingosine) may be the enzyme responsible for producing sphingosine to prime synaptic vesicles for docking with the plasma membrane. Indeed, ceramidase has been shown to be an important regulator of 
synaptic vesicle exocytosis (Rohrbough et al. 2004). However, there is also evidence that sphingosine kinase-1 plays a role in regulating neurotransmitter release by converting sphingosine to sphingosine 1-phosphate (S1P) presumably through paracrine actions at the presynaptic terminal dependent on the S1P 1 receptor (Kajimoto et al. 2007; Bajjalieh et al. 1989). The sphingomyelin phosphodiesterase neutral sphingomyelinase 2 has also been implicated in regulating neurotransmitter release by rapidly creating ceramide to promote the fusion of vesicles with the plasma membrane (Numakawa et al. 2003; Jeon et al. 2005; Blochl and Thoenen 1996).

\section{Neurotherapeutics that Target Sphingolipid Metabolism}

Our knowledge of the roles that sphingolipids play in regulating neuronal, glial and immunological functions is increasing at a rapid pace. So too is our understanding of how perturbations in sphingolipid metabolism contribute to neurodegeneration in a variety of neurodegenerative conditions. Since enzymes that regulate sphingolipid metabolism are natural targets for the development of traditional small molecule therapeutics, it is not surprising that a number of candidate therapeutics have been developed and tested that target pathways for ceramide production, glycolipid pathways and receptors for sphingosine 1phosphate (among others). Based on findings from current and ongoing studies highlighted in this special issue of NeuroMolecular Medicine, it is seems likely that future therapies for treating neurodegeneration will include agents that modulate sphingolipid metabolism, either as primary therapeutics or in combination with other drugs.

\section{References}

Abulrob A, Tauskela JS, Mealing G, Brunette E, Faid K, Stanimirovic D. Protection by cholesterolextracting cyclodextrins: A role for N-methyl-D-aspartate receptor redistribution. Journal of Neurochemistry. 2005; 92:1477-1486. [PubMed: 15748165]

Assaife-Lopes N, Sousa VC, Pereira DB, Ribeiro JA, Chao MV, Sebastiao AM. Activation of adenosine A2A receptors induces TrkB translocation and increases BDNF-mediated phospho-TrkB localization in lipid rafts: Implications for neuromodulation. Journal of Neuroscience. 2010; 30:8468-8480. [PubMed: 20573894]

Bajjalieh SM, Martin TF, Floor E. Synaptic vesicle ceramide kinase. A calcium-stimulated lipid kinase that co-purifies with brain synaptic vesicles. Journal of Biological Chemistry. 1989; 264:1435414360. [PubMed: 2547795]

Bandaru VV, McArthur JC, Sacktor N, Cutler RG, Knapp EL, Mattson MP, et al. Associative and predictive biomarkers of dementia in HIV-1-infected patients. Neurology. 2007; 68:1481-1487. [PubMed: 17470750]

Bandaru VV, Troncoso J, Wheeler D, Pletnikova O, Wang J, Conant K, et al. ApoE4 disrupts sterol and sphingolipid metabolism in Alzheimer's but not normal brain. Neurobiology of Aging. 2009; 30:591-599. [PubMed: 17888544]

Besshoh S, Bawa D, Teves L, Wallace MC, Gurd JW. Increased phosphorylation and redistribution of NMDA receptors between synaptic lipid rafts and post-synaptic densities following transient global ischemia in the rat brain. Journal of Neurochemistry. 2005; 93:186-194. [PubMed: 15773918]

Blochl A, Thoenen H. Localization of cellular storage compartments and sites of constitutive and activity-dependent release of nerve growth factor (NGF) in primary cultures of hippocampal neurons. Molecular and Cellular Neurosciences. 1996; 7:173-190. [PubMed: 8726102]

Blood PD, Voth GA. Direct observation of Bin/amphiphysin/Rvs (BAR) domain-induced membrane curvature by means of molecular dynamics simulations. Proceedings of the National Academy of Sciences of the United States of America. 2006; 103:15068-15072. [PubMed: 17008407] 
Brann AB, Scott R, Neuberger Y, Abulafia D, Boldin S, Fainzilber M, et al. Ceramide signaling downstream of the p75 neurotrophin receptor mediates the effects of nerve growth factor on outgrowth of cultured hippocampal neurons. Journal of Neuroscience. 1999; 19:8199-8206. [PubMed: 10493721]

Bryant MR, Marta CB, Kim FS, Bansal R. Phosphorylation and lipid raft association of fibroblast growth factor receptor-2 in oligodendrocytes. Glia. 2009; 57:935-946. [PubMed: 19053057]

Coogan AN, O'Neill LA, O'Connor JJ. The P38 mitogen-activated protein kinase inhibitor SB203580 antagonizes the inhibitory effects of interleukin-1beta on long-term potentiation in the rat dentate gyrus in vitro. Neuroscience. 1999; 93:57-69. [PubMed: 10430470]

Darios F, Wasser C, Shakirzyanova A, et al. Sphingosine facilitates SNARE complex assembly and activates synaptic vesicle exocytosis. Neuron. 2009; 62:683-694. [PubMed: 19524527]

Davis CN, Tabarean I, Gaidarova S, Behrens MM, Bartfai T. IL-1beta induces a MyD88-dependent and ceramide-mediated activation of Src in anterior hypothalamic neurons. Journal of Neurochemistry. 2006; 98:1379-1389. [PubMed: 16771830]

Day CA, Kenworthy AK. Tracking microdomain dynamics in cell membranes. Biochimica et Biophysica Acta. 2009; 1788:245-253. [PubMed: 19041847]

Delint-Ramirez I, Fernandez E, Bayes A, Kicsi E, Komiyama NH, Grant SG. In vivo composition of NMDA receptor signaling complexes differs between membrane subdomains and is modulated by PSD-95 and PSD-93. Journal of Neuroscience. 2010; 30:8162-8170. [PubMed: 20554866]

Fasano C, Miolan JP, Niel JP. Modulation by C2 ceramide of the nicotinic transmission within the coeliac ganglion in the rabbit. Neuroscience. 2003; 116:753-759. [PubMed: 12573717]

Frank C, Giammarioli AM, Pepponi R, Fiorentini C, Rufini S. Cholesterol perturbing agents inhibit NMDA-dependent calcium influx in rat hippocampal primary culture. FEBS Letters. 2004; 566:25-29. [PubMed: 15147862]

Fullekrug J, Simons K. Lipid rafts and apical membrane traffic. Annals of the New York Academy of Sciences. 2004; 1014:164-169. [PubMed: 15153431]

Furukawa K, Mattson MP. The transcription factor NF-kappaB mediates increases in calcium currents and decreases in NMDA- and AMPA/kainate-induced currents induced by tumor necrosis factoralpha in hippocampal neurons. Journal of Neurochemistry. 1998; 70:1876-1886. [PubMed: 9572271]

Furuya S, Mitoma J, Makino A, Hirabayashi Y. Ceramide and its interconvertible metabolite sphingosine function as indispensable lipid factors involved in survival and dendritic differentiation of cerebellar Purkinje cells. Journal of Neurochemistry. 1998; 71:366-377. [PubMed: 9648886]

Harlan JE, Hajduk PJ, Yoon HS, Fesik SW. Pleckstrin homology domains bind to phosphatidylinositol-4, 5-bisphosphate. Nature. 1994; 371:168-170. [PubMed: 8072546]

Haughey NJ, Cutler RG, Tamara A, McArthur JC, Vargas DL, Pardo CA, et al. Perturbation of sphingolipid metabolism and ceramide production in HIV-dementia. Annals of Neurology. 2004; 55:257-267. [PubMed: 14755730]

Hering H, Lin CC, Sheng M. Lipid rafts in the maintenance of synapses, dendritic spines, and surface AMPA receptor stability. Journal of Neuroscience. 2003; 23:3262-3271. [PubMed: 12716933]

Hirao M, Sato N, Kondo T, Yonemura S, Monden M, Sasaki T, et al. Regulation mechanism of ERM (ezrin/radixin/moesin) protein/plasma membrane association: Possible involvement of phosphatidylinositol turnover and Rho-dependent signaling pathway. Journal of Cell Biology. 1996; 135:37-51. [PubMed: 8858161]

Inokuchi J, Mizutani A, Jimbo M, et al. A synthetic ceramide analog (L-PDMP) up-regulates neuronal function. Annals of the New York Academy of Sciences. 1998; 845:219-224. [PubMed: 9668355]

Ito A, Horigome K. Ceramide prevents neuronal programmed cell death induced by nerve growth factor deprivation. Journal of Neurochemistry. 1995; 65:463-466. [PubMed: 7790893]

Jeon HJ, Lee DH, Kang MS, Lee MO, Jung KM, Jung SY, et al. Dopamine release in PC12 cells is mediated by $\mathrm{Ca}(2$ ?)-dependent production of ceramide via sphingomyelin pathway. Journal of Neurochemistry. 2005; 95:811-820. [PubMed: 16135082] 
Kajimoto T, Okada T, Yu H, Goparaju SK, Jahangeer S, Nakamura S. Involvement of sphingosine-1phosphate in glutamate secretion in hippocampal neurons. Molecular and Cellular Biology. 2007; 27:3429-3440. [PubMed: 17325039]

Kutateladze TG, Ogburn KD, Watson WT, de Beer T, Emr SD, Burd CG, et al. Phosphatidylinositol 3phosphate recognition by the FYVE domain. Molecular Cell. 1999; 3:805-811. [PubMed: 10394369]

Levental I, Grzybek M, Simons K. Greasing their way: Lipid modifications determine protein association with membrane rafts. Biochemistry. 2010; 49:6305-6316. [PubMed: 20583817]

Liu Y, Fisher DA, Storm DR. Analysis of the palmitoylation and membrane targeting domain of neuromodulin (GAP-43) by site-specific mutagenesis. Biochemistry. 1993; 32:10714-10719. [PubMed: 8399217]

Low MG. Glycosyl-phosphatidylinositol: A versatile anchor for cell surface proteins. The FASEB Journal. 1989; 3:1600-1608.

Magee AI, Gutierrez L, Marshall CJ, Hancock JF. Targeting of oncoproteins to membranes by fatty acylation. Journal of Cell Science. Supplement. 1989; 11:149-160. [PubMed: 2533203]

Medkova M, Cho W. Interplay of C1 and C2 domains of protein kinase C-alpha in its membrane binding and activation. Journal of Biological Chemistry. 1999; 274:19852-19861. [PubMed: 10391930]

Numakawa T, Nakayama H, Suzuki S, et al. Nerve growth factor-induced glutamate release is via p75 receptor, ceramide, and $\mathrm{Ca}(2$ ?) from ryanodine receptor in developing cerebellar neurons. Journal of Biological Chemistry. 2003; 278:41259-41269. [PubMed: 12902347]

Owen DM, Williamson D, Rentero C, Gaus K. Quantitative microscopy: Protein dynamics and membrane organisation. Traffic. 2009; 10:962-971. [PubMed: 19416480]

Ping SE, Barrett GL. Ceramide can induce cell death in sensory neurons, whereas ceramide analogues and sphingosine promote survival. Journal of Neuroscience Research. 1998; 54:206-213. [PubMed: 9788279]

Rohrbough J, Rushton E, Palanker L, Woodruff E, Matthies HJ, Acharya U, et al. Ceramidase regulates synaptic vesicle exocytosis and trafficking. Journal of Neuroscience. 2004; 24:77897803. [PubMed: 15356190]

Santagata S, Boggon TJ, Baird CL, Gomez CA, Zhao J, Shan WS, et al. G-protein signaling through tubby proteins. Science. 2001; 292:2041-2050. [PubMed: 11375483]

Stahelin RV. Lipid binding domains: More than simple lipid effectors. Journal of Lipid Research. 2009; 50(Suppl):S299-S304. [PubMed: 19008549]

Stahelin RV, Long F, Peter BJ, Murray D, De Camilli P, McMahon HT, et al. Contrasting membrane interaction mechanisms of AP180 N-terminal homology (ANTH) and epsin N-terminal homology (ENTH) domains. Journal of Biological Chemistry. 2003; 278:28993-28999. [PubMed: 12740367]

Stokoe D, Macdonald SG, Cadwallader K, Symons M, Hancock JF. Activation of Raf as a result of recruitment to the plasma membrane. Science. 1994; 264:1463-1467. [PubMed: 7811320]

Swartz KJ. Sensing voltage across lipid membranes. Nature. 2008; 456:891-897. [PubMed: 19092925]

Tabarean IV, Korn H, Bartfai T. Interleukin-1beta induces hyperpolarization and modulates synaptic inhibition in preoptic and anterior hypothalamic neurons. Neuroscience. 2006; 141:1685-1695. [PubMed: 16777343]

Thelen M, Rosen A, Nairn AC, Aderem A. Regulation by phosphorylation of reversible association of a myristoylated protein kinase C substrate with the plasma membrane. Nature. 1991; 351:320-322. [PubMed: 2034276]

Wheeler D, Knapp E, Bandaru VV, Wang Y, Knorr D, Poirier C, et al. Tumor necrosis factor-alphainduced neutral sphingomyelinase- 2 modulates synaptic plasticity by controlling the membrane insertion of NMDA receptors. Journal of Neurochemistry. 2009; 109:1237-1249. [PubMed: 19476542]

Yang SN. Ceramide-induced sustained depression of synaptic currents mediated by ionotropic glutamate receptors in the hippocampus: An essential role of postsynaptic protein phosphatases. Neuroscience. 2000; 96:253-258. [PubMed: 10683565] 
Yin X, Zafrullah M, Lee H, Haimovitz-Friedman A, Fuks Z, Kolesnick R. A ceramide-binding C1 domain mediates kinase suppressor of ras membrane translocation. Cellular Physiology and Biochemistry. 2009; 24:219-230. [PubMed: 19710537] 